Recht und Strafrecht, da es als unzumutbar empfunden wurde, wenn der Bürger in den beiden Teilen des Landes einer unterschiedlichen Ordnungs- oder Strafgewalt unterlegen hätte (S. 88). Sonst erfährt man über die Überlegungen im Rechtssetzungsprozeß nur wenig, wenn man sich nicht mit der Auskunft begnügen will, das materielle italienische Strafrecht sei übernommen worden, weil die Mehrzahl der Regierungsmitglieder und Beamten italienisch erzogen worden seien (S. 45), und das englische Prozeßrecht, weil es während der Zeit der britischen Militärverwaltung großen Eindruck gemacht habe (S. 46). Es ist denkbar, daß die Kommission weitergehende Überlegungen tatsächlich nicht angestellt hat. Dabei ist ihr zugute zu halten, daß sie unter einem großen Zeitdruck arbeiten mußte (S. 45). Bedauerlich wäre es dennoch, da der Fall Somalia die einmalige Chance geboten hätte, die Frage zu untersuchen, ob eins der europäischen Rechtssysteme sich besser für Modernisierung und Entwicklung einsetzen läßt, oder ob diese Aufgabe von einer Synthese beider Systeme am besten gelöst werden kann. Für diese Frage, die vielleicht die Kernfrage der Erforschung des Rechts der afrikanischen Entwicklungsländer ist, bietet das Buch Material, ohne zu ihrer Lösung Wesentliches beizutragen.

Brun-Otto Bryde

\section{James D. Cochrane}

\section{The Politics of Regional Integration}

The Central American Case

Tulane Studies in Political Science, Volume XII

Tulane University, New Orleans, 1969

Martinus Nijhoff, The Hague, 1969

225 Seiten, Preis hfl. 14.40.

Die nach dem zweiten Weltkrieg in vielen Regionen der Welt aufgetretenen Bestrebungen, durch Zusammenschluß benachbarter Länder größere Entwicklungserfolge $\mathrm{zu}$ erzielen, haben schon früh auch Lateinamerika ergriffen. In diesem Bereich verdient der bisher weniger beachtete Zentralamerikanische
Gemeinsame Markt insofern besondere Beachtung, als er im Gegensatz zu anderen Zusammenschlüssen bereits konkrete Ergebnisse erzielen konnte, die über eine bloße Förderung des intrazonalen Handels hinausgehen und den Weg weisen $z u$ echten Integrationen. Dem Werdegang dieser Bemühungen nachzugehen, ist der Zweck dieser interessanten und beachtlichen Neuerscheinung.

Wirtschaftliche Integrationen pflegen darunter zu leiden, daß die in ihr ruhenden politischen Fragen ausgeklammert werden. Cochrane geht davon aus, daß alle regionalen Zusammenschlüsse letzten Endes politischer Natur sind. Dies trifft auf Zentralamerika von vornherein deshalb zu, weil historische Grundlagen für eine politische Einigung gegeben sind, die vom Autor auch in seine Betrachtung einbezogen werden. Die fünf Teilnehmerländer (Costa Rica, Guatemala, El Salvador, Honduras, Nicaragua mit (1965) rd. 12 Mill. Einwohnern) waren bis 1840 in einer Union vereinigt. Wenn sie sich auch seit dieser Zeit auseinandergelebt haben, was sich zu offenen Konflikten und Animositäten steigern kann (siehe Konflikt Honduras-El Salvador im Jahre 1969) so geistert doch trotz aller Gegensätze das Ideal einer politischen Union als bisher nicht verwirklichter Wunschtraum in Köpfen von Zentralamerikanern. Jedenfalls unterscheidet sich die zentralamerikanische Integration von anderen dadurch, daß irgendwie doch das Politische mindestens im Untergrunde als Stimulans für die wirtschaftliche Kooperation mitwirkt. Der auch in diesen Ländern wachsende Nationalismus und das Empfinden für ,independencia“ treffen sich mit dem Gefühl, daß diese fünf Länder auf der mittelamerikanischen Landbrücke, jedes für sich zu schwach und klein, aber insgesamt eine räumliche Einheit bilden, wirtschaftlich und politisch aufeinander angewiesen sind und auch ihre nationalen Ziele nur durch gemeinsames Vorgehen erfolgreich anstreben können. Wirtschaftliche Integrationen sind unlöslich verbunden mit den sozialen Problemen, mit denen 
sich Cochrane ausführlich mit sympathischer Offenheit befaßt. Die traditionellen Feudalstrukturen, wovon sich auch Costa Rica nur gradmäßig unterscheidet, mit dem "wide gap" $z$ wischen reich und arm sind auch in Zentralamerika heute noch ebenso beherrschende Basisprobleme wie das Büdnnis der obersten Klasse mit der jeweiligen Regierung und der Fortdauer kolonialer Ausbeutung. "The full story" würde meist nicht erzählt, bemerkte Cochrane; die obersten Klassen hätten sehr wenig getan für die Entwicklung dieser Länder, oft fehle es ihnen an sozialem Verständnis. Soziale Unruhen sind daher unvermeidlich, auch sie werden den Fortgang der Integration beeinflussen! Ohne dieser sozialen ebenso wie der politischen Problematik auszuweichen, schildert der Verfasser eingehend Grundlagen, Motive, Entwicklung und Ergebnisse der seitherigen Wirtschafts-Integration, die nach bilateralen Vorläufern aus den 50er Jahren endgültig mit dem Vertrage vom Dezember 1960 (gültig ab 3. 6. 61) zwischen El Salvador, Honduras, Guatemala und Nicaragua einsetzte; Costa Rica trat 1963 hinzu. Auf die Zielsetzung hatten die Ideen der CEPAL (Comisión Económica para América Latina) groEinfluß. Man ging von vornherein über eine bloße Freihandelszone hinaus. Der Abbau der Binnenzölle war 1966 bereits zu 95 Prozent erreicht, der gemeinsame Außenzoll im Juni 1966 schon zu 98 Prozent verwirklicht. Mit Erfolg bemühte man sich um eine Vereinheitlichung des Verkehrswesens. Die 1963 gegründete Zentralamerikanische Entwicklungsbank ist ein wesentliches Element der Förderung; auf Basis des CA-Dollar erfolgt die Verrechnung des Intrazonenhandels. Das alles sind Tatbestände, die die verhältnismäßig hohen und sicherlich anfangs kaum erwarteten Erfolge im Handelsaustausch und im beginnenden industriellen Aufbau erklären. So hat sich der intrazonale Handelsaustausch von 1960 mit 32,7 Mill. US $\$$ bis 1968 auf 259,2 Mill. US $\$$ erhöht, oder prozentual: von 6,4 Prozent - 1960 auf 15,6 Prozent - 1965. Dabei ist vor allem der Export von Fertig- produkten von 1960 auf 1965 um das 6 fache gestiegen (15,5 Mill. US $\$$ auf 98 Mill. US \$). Diese Zahlen deuten auf eine fortschreitende Diversifizierung von Wirtschaft und Außenhandel hin, im Gegensatz zu dem Zustand vorher, der durch weitgehend gleiche und einseitige Agrarproduktion und eine schwache, nur für den nationalen Konsum arbeitende Industrie gekennzeichnet war. In der Agrarwirtschaft ist ein wesentlicher und notwendiger Fortschritt zur Modernisierung noch kaum eingetreten. Es gilt das traditionelle paternalistische Feudalsystem mit der Bindung der analphabetischen und oft unterernährten Landarbeiter an die $\mathrm{Ha}$ cienda; die Landwirtschaft beschäftigt zwischen 50 und 80 Prozent der Arbeitskräfte. Das für die Mehrzahl dieser Länder typische Latifundiensystem, vor allem auf den Export von Kaffee, Baumwolle und Bananen ausgerichtet, besteht generell weiter - daneben die Minifundien mit ihrer Subsistenzwirtschaft. Wenn auch eine Rationalisierung und Modernisierung der Agrarwirtschaft notwendig ist, so wird andererseits kaum mit einer Ausweitung des Exportes und Erschließung neuer Märkte für die traditionellen Produkte zu rechnen sein. Immerhin hat sich die Agrarproduktion bereits verdoppelt.

Cochrane verwendet leider nur Zahlen bis 1965, so daß die allerneueste Entwicklung, in der sich Schwierigkeiten zeigten, nicht sichtbar wird. Immerhin sind die bisherigen Ergebnisse beachtlich, auch wenn es noch an einer allgemeinen „integration ideology" fehlt, und damit an genügender Initiative aus der Bevölkerung heraus, an der „popular dynamic". Wesentlich getragen wird eine solche Integration zunächst mehr von den "técnicos“, der „integration mafia".

Der ZAGM ist sicherlich kein generell gültiges Modell - immerhin kann er wertvolle Einsichten vermitteln. Cochrane faßt die Integration richtigerweise als Prozeß auf, der auf bestimmten Sektoren Erfolge erzielen konnte, der aber wegen der vielen noch offenen und schwerwiegenden Basisprobleme in 
seinem weiteren Werdegang vieles offen läßt. Auch das Ausland kann durch verständnisvolle Förderung viel beitragen, was vor allem auch für die USA zu gelten hat, für die Zentralamerika stets ein wichtiges Interessengebiet war. Immerhin sei, generell gesehen, vieles erreicht, was schon fast an eine wirtschaftliche und sogar an eine politische Revolution in Zentralamerika heranreiche. Hierin kann man dem Verfasser nur zustimmen; für die ausführliche und nüchterne Erörterung aller Probleme muß man dankbar sein.

Friedrich Wehner

James L. Payne

Patterns of Conflict in Colombia

New Haven and London Yale University Press, 1968, XV + 358 Seiten.

Dieses Buch hat sich zum Ziel gesetzt „to explain political conflict in Colombia through an examination of political motives and the context in which these motives find expression" (4). Trotzdem scheint es dem Verfasser weithin weniger auf Empirie als solche denn auf die Verifizierung abstrakter Thesen anzukommen, so daß man nicht immer sicher ist, ob man es mit Referat oder mit Deduktion zu tun hat. Abgesehen hiervon handelt es sich um eine politologisch-soziologische Beschreibung der staatlichen Institutionen, Zustände und vor allem der Motivationen der auf diesen Bühnen sich bewegenden Akteure. Zweierlei mag hieraus als verfassungstheoretisch bedeutsam herausgehoben werden.

Nicht allzusehr überrascht die Feststellung, daß es der Politik und den Politikern nicht um irgendeine Sache, sondern um die Person, ihren Status und ihr Prestige geht (VII; 241; 244; 248 und öfter). Die Parteien können sich folgerichtig nicht durch Programme unterscheiden; hierfür bleibt lediglich übrig eine Differenzierung nach der Strategie der persönlichen Statuserlangung und -behauptung, also praktisch eine Minimisierung der Verschiedenheit.
Unter solchen Umständen kann das Parlament nichts anderes sein als eine unpolitische Veranstaltung (246): „We think Congress is for speeches not votes" (ein kolombianischer Abgeordneter; vgl. S. 238). Eine solche Auffassung macht es unvermeidlich, die eigentlichen Entscheidungen an die Exekutive zu delegieren (257).

Kolumbien zeigt, daß eine am persönlichen Status orientierte „Politik“ Gewalt, und zwar sogar extreme Gewalt nicht ausschließt, ja sogar zu begünstigen scheint. Der Bürgerkrieg mag hier die Fortsetzung der Innen,politik“ mit anderen Mitteln sein. Man schätzt, daß der letzte von ihnen (1949-1953) insgesamt 134820 Menschenleben gefordert hat. Es ist die Gesellschaft selbst, die ein Heilmittel gegen diese seit 1826 schwelende Krisis (314) gefunden hat. Es ist die der "Frente Nacional“ (177) - eine Art von Friedensschluß zwischen den beiden großen Parteien, der beider Parität in Parlament und Regierung sowie Alternation der Präsidentschaft vorsieht. Durch Volksabstimmung ist er zum Bestandteil der Verfassung erhoben worden. Diese Lösung hat sich bewährt (315). Mit um so größerer Besorgnis sieht man daher dem Jahre 1974 entgegen, in dem dieser Vertrag abläuft.

Herbert Krüger

\section{ERnest A. Duff}

Agrarian Reform in Colombia

Praeger Special Studies in International Economics and Development

Frederick A. Praeger, New York 1968, XI, 240 S.,

Fassung des Agrarreform-Gesetzes 135 von 1961, Bibliographie, US $\$ 14,00$.

Agrarreformen stellen ein neuralgisches Problem in den meisten Ländern Lateinamerikas dar. Größtenteils als fortschrittshemmende Erbschaft hispanischer Kolonisierungspolitik verstanden, kommt der höchst ungleichen Verteilung des Landbesitzes nicht nur eine soziale und ökonomische Bedeutung $\mathrm{zu}$, sondern läßt aufgrund der gegebenen Gesellschaftsstrukturen aus allen Re- 